\title{
Performance of Long Ladders for the LHCb Silicon Tracker*
}

\author{
M. Agari, C. Bauer, J. Blouw, W. Hofmann, K.T.Knöpfle, S.Löchner, \\ F. Maciuc, M. Schmelling, N. Smale, B. Schwingenheuer, H. Voss \\ Max Planck Institut für Kernphysik \\ Saupfercheckweg 1, 69117 Heidelberg, Germany \\ V. Pugatch, Y. Pylypchenko \\ National Academy of Science \\ Institute for Nuclear Research, 03680 Kiev, Ukraine
}

A. Bay, M.-O. Bettler, B. Carron, P. Fauland, R. Frei, S. Jimenez-Otero, L. Nicolas, A. Perrin, O. Schneider, M.T. Tran, J. van Hunen, K.Vervink, A. Vollhardt

LPHE, EPFL Lausanne Bât. des Sciences Physiques, 1015 Dorigny, Switzerland

B. Adeva, D. Esperante, C. Lois, E. Perez Trigo, M. Plo Casasus, P. Vazquez Universidad de Santiago de Compostela

Campus Universitario Sur, 15706 Santiago de Compostela, Spain

R.P. Bernhard, R. Bernet, J. Gassner, S. Koestner, F. Lehner, M. Needham, T. Sakhelashvili, S. Steiner, O. Steinkamp, U. Straumann, D. Volyanskyy, A. Wenger Physik-Institut der Universität Zürich Winterthurerstr. 190, 8057 Zürich, Switzerland

December 24, 2005

\begin{abstract}
The LHCb Silicon Tracker uses detector ladders with readout strips with an effective length of up to $36 \mathrm{~cm}$. Kapton interconnect cables of up to $54 \mathrm{~cm}$ in length are employed in between silicon sensors and front-end readout hybrids. Fast front-end readout electronics with a shaping time of around 25 ns are employed to avoid pile-up of events from consecutive LHC bunch crossings. An extensive measurement program as well as simulations have been carried out to study the expected noise performance of these detectors.
\end{abstract}

\section{Introduction}

The Silicon Tracker is part of the LHCb tracking system [1]. It comprises two detectors, both of which use silicon microstrip detectors. The Trigger Tracker (TT) is a four-layer planar tracking station that is located upstream of the dipole magnet of the LHCb spectrometer and covers the full acceptance of the experiment. The Inner Tracker (IT) covers the region close to the LHC beam-pipe in three four-layer tracking stations downstream of the magnet.

${ }^{*}$ Presented at the 14th International Workshop on Vertex Detectors, Chuzenji Lake, Nikko, Japan, November 7-11, 2005

Proceedings submitted for publication in Nuclear Instruments and Methods A 
Taken together, TT and IT cover a sensitive surface of about $14 \mathrm{~m}^{2}$. An important design goal in the development of the detector was to cover this surface with the smallest possible number of readout channels in order to keep the cost of the detector at an acceptable level.

Detector ladders for the IT are constructed from either a single $320 \mu \mathrm{m}$ thick silicon strip sensor or two ganged silicon sensors of $410 \mu \mathrm{m}$ in thickness. Both types of sensors have a strip length of $108 \mathrm{~mm}$, a pitch of $198 \mu \mathrm{m}$ and an implant width of $50 \mu \mathrm{m}$.

The TT employs ladders that are constructed from seven $500 \mu$ m thick silicon sensors ${ }^{1}$ with a strip length of $94 \mathrm{~mm}$, a strip pitch of $183 \mu \mathrm{m}$ and an implant width of $45 \mu \mathrm{m}$. Each TT ladder is electronically split into several readout sectors. Ladders in the outer part of the detector are split into an outer four-sensor long readout sector and an inner three-sensor long sector, whereas ladders close to the beampipe have the three innermost sensors segmented into a two-sensor long readout sector and a readout sector consisting of a single sensor. In order to minimise the material budget inside the acceptance of the experiment, the front-end readout hybrids for all readout sectors are located at one end of the ladder, outside of the acceptance. The inner readout sectors are connected to their hybrids via flexible Kapton interconnect cables. These cables have a length of $37.6 \mathrm{~cm}$ for the three-sensor long and two-sensor long sectors and a length of $56.4 \mathrm{~cm}$ for the one-sensor sector. They carry thin gold-plated copper strips with a width of $16 \mu \mathrm{m}$ and a pitch of $112 \mu \mathrm{m}$. A copper-mesh on the backplane of the $100 \mu \mathrm{m}$ thick Kapton substrate serves as return path for detector signals and reduces the risk of pick-up noise.

Monte-Carlo studies have demonstrated that the chosen detector layout results in low occupancies of a few percent or less and gives an adequate spatial resolution of about $50 \mu \mathrm{m}$.

Both TT and IT are read out using the Beetle [3] readout chip. The front-end of the chip has been optimised to deal with the large load capacitances expected for the Silicon Tracker. A programmable parameter called $V_{\mathrm{fs}}$ permits to tune the output pulse shape of the frontend amplifiers within certain limits. The most relevant pulse-shape parameter for operation at the LHC is the remaining signal amplitude at the time of the next LHC bunch crossing - $25 \mathrm{~ns}$ after the maximum of the pulse. Monte-Carlo studies of the tracking performance have shown that remainders of $30 \%$ and $50 \%$ of the signal amplitude can be accepted for IT and TT, respectively. Measurements on prototype detectors have demonstrated that these requirements can be met for a setting of $V_{\mathrm{fs}}=400 \mathrm{mV}$. All results presented below were obtained for this setting. If required, the quoted signal-to-noise performances can be improved - albeit at the expense of higher signal remainders — by operating at higher values of $V_{\mathrm{fs}}$.

\section{Noise performance}

In view of the large strip capacitances and the fast pulse shapes required for operation at the LHC, the signal-to-noise performance of the detectors was a major concern in the design phase of the Silicon Tracker. An extensive R\&D program, including measurements in an infra-red laser test stand [4] and in a $120 \mathrm{GeV} \pi^{-}$beam at CERN [5], was carried out to determine the required sensor thickness for the different parts of the Silicon Tracker and to demonstrate that an acceptable signal-to-noise performance could be achieved. Various prototype detectors, with effective readout strip lengths of $108 \mathrm{~mm}$ up to $324 \mathrm{~mm}$, were included in these tests. They were constructed from silicon sensors of $320 \mu \mathrm{m}$ to $500 \mu \mathrm{m}$ in thickness, with strip pitches between $183 \mu \mathrm{m}$ and $228 \mu \mathrm{m}$, and with ratios of implant width over strip pitch of 0.25 to 0.35 . In particular, one of the tested ladder consisted of three CMS-OB2 sensors and a

\footnotetext{
${ }^{1}$ These sensors are identical to the OB2-type sensors used in the outer barrel of the CMS Silicon Tracker [2].
} 


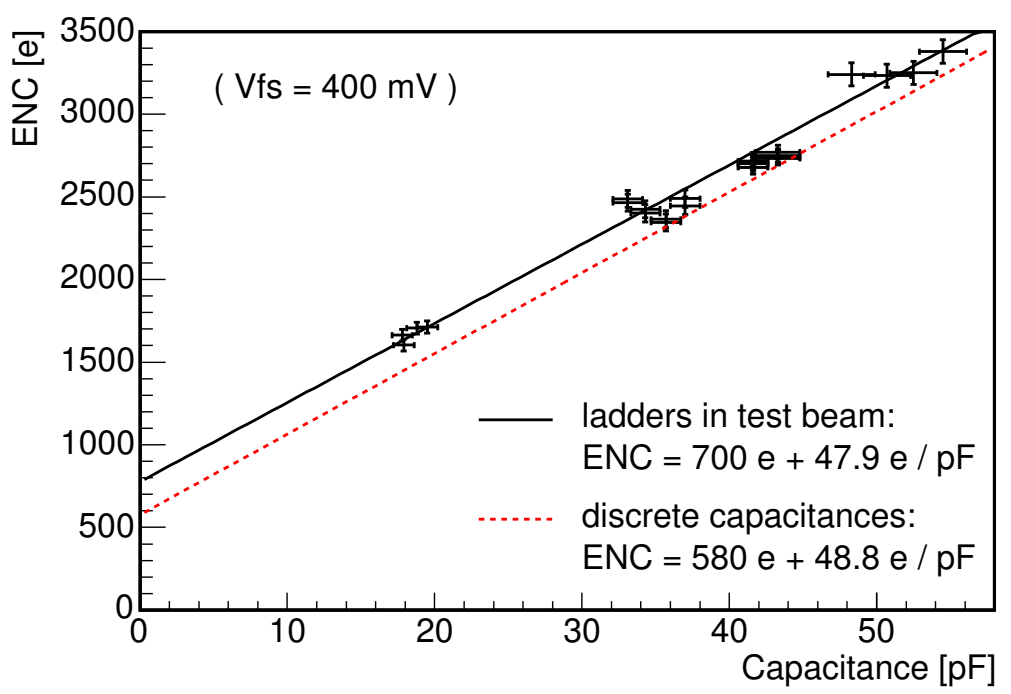

Figure 1: ENC obtained in test-beam measurements as a function of the measured total strip capacitance of the tested prototype ladders.

$40 \mathrm{~cm}$ long Kapton interconnect cable.

As input for a detailed noise study, the total capacitance, the serial resistance and the inductance of the readout strips were determined for each tested ladder. Total strip capacitances were measured on each ladder and calculated in a 2D Maxwell [6] simulation, the results agreeing within $10 \%$. The serial resistance of the readout strips was both measured and calculated, again with consistent results. Finally, the strip inductance was calculated in a 2D Maxwell simulation.

The measured noise performance from beam-tests is summarised in Figure 1, in which the equivalent noise charge (ENC) for each prototype ladder is shown as a function of the total strip capacitance. A linear dependence is observed and the slope of a line fitted to the data agrees well with that obtained in test-bench measurements, in which discrete load capacitances were attached to the Beetle inputs ${ }^{2}$. There is no indication for any significant additional noise contribution from the serial resistance of the long readout strips. The linear dependence of the noise on the detector capacitance permits to calculate the expected noise performance for all types of detector ladders employed in the Silicon Tracker. With the chosen sensor thicknesses, most probable signal-to-noise ratios in excess of 12:1 can be expected for all ladders and readout sectors.

In addition to these measurements, a Spice simulation was carried out in which the long readout strips were modelled as extended $R L C$-devices with ten discrete $R L C$-elements per $\mathrm{cm}$ strip-length [7]. As an example, the calculated noise spectrum obtained for the threesensor ladder with interconnect cable is shown in Figure 2. Strip capacitance, resistance and inductance were calculated and simulated individually for the readout strips on the silicon sensors and for the strip lines on the Kapton cable. As expected, white noise from the Beetle input FET (modelled by an equivalent noise resistor of $68 \Omega$ ) dominates at low frequencies. The contribution from the readout strips becomes sizeable at frequency above approximately $3 \mathrm{MHz}$. Above $100 \mathrm{MHz}$ - the lowest Eigenfrequency of the $R L C$ network - a resonating

\footnotetext{
${ }^{2}$ The constant term is somewhat larger for the test beam results than for the test-bench measurements. This effect was not studied in detail but is assumed to be due to unidentified external noise sources in the test-beam setup.
} 


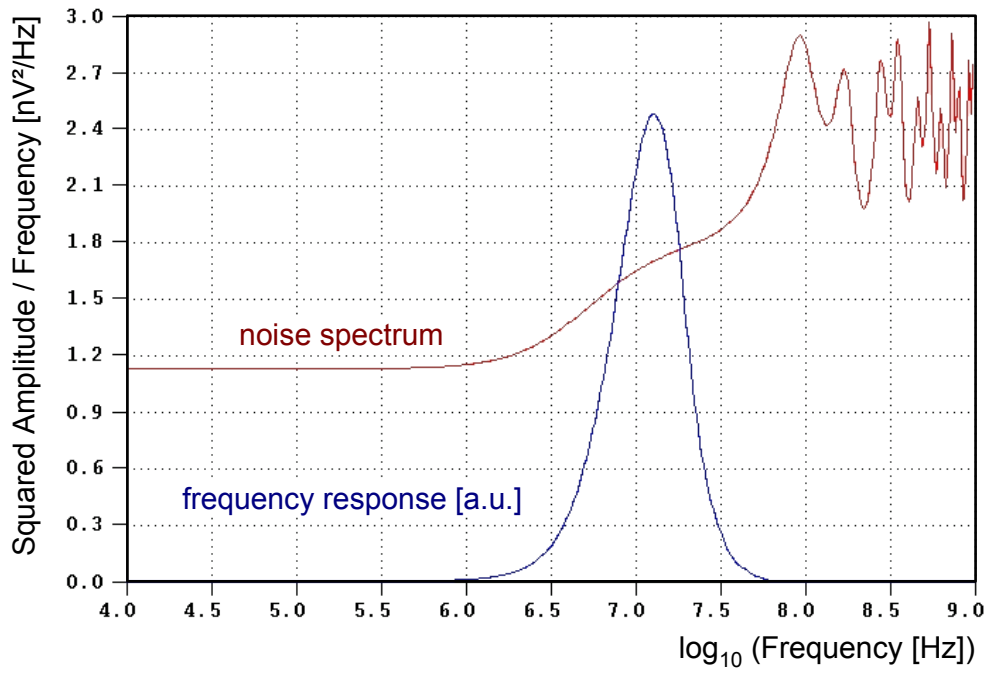

Figure 2: Calculated noise spectrum for the simulated detector ladder, and Beetle frequency response function. For details of the simulation, see [7].

behaviour is observed. Some uncertainty in the result of the simulation stems from poorly known parameters, such as the inductivity of the strip lines and the input impedance of the Beetle chip.

Also shown in Figure 2 is the calculated frequency response function of the Beetle frontend amplifier, obtained from a Laplace transformation of the measured response function for a fast input current pulse. The total noise can be calculated as the convolution of this frequency response function with the squared noise spectrum. The frequency response function of the Beetle peaks at around $10 \mathrm{MHz}$, in the rising part of the noise spectrum. Therefore, the calculation of the total noise suffers from systematic uncertainties due to the uncertainties in the calculation of the noise spectrum mentioned above. For the default parameters of the simulation, a total noise of about $3900 \mathrm{e}^{-}$is calculated for the ladder, compared to a noise of about $3300 \mathrm{e}^{-}$for an equivalent discrete load capacitance of $57 \mathrm{pF}$. This result indicates an $18 \%$ increase of the noise due to the strip resistance on the ladder, albeit with a significant systematic uncertainty.

\section{Signal shape}

Another aspect that was studied in the Spice simulation was the pulse shape of the detector signal. In Figure 3, calculated pulse shapes at the input of the Beetle amplifier are shown, again for the example of the ladder consisting of three CMS-OB2 sensors with interconnect cable. In addition, the pulse shape for a discrete capacitance of $57 \mathrm{pF}$ is shown for comparison. The pulse shapes for the ladder show some structure due to signal reflections at the end of the ladder and at the interface between sensor and interconnect cable. These structures are, however, washed out by the charge integration in the Beetle amplifiers. This is illustrated in Figure 4, which shows the same signals at the output of the Beetle front-end amplifiers. Also shown in this figure is the measured pulse shape for the ladder, obtained in an infra-red laser 


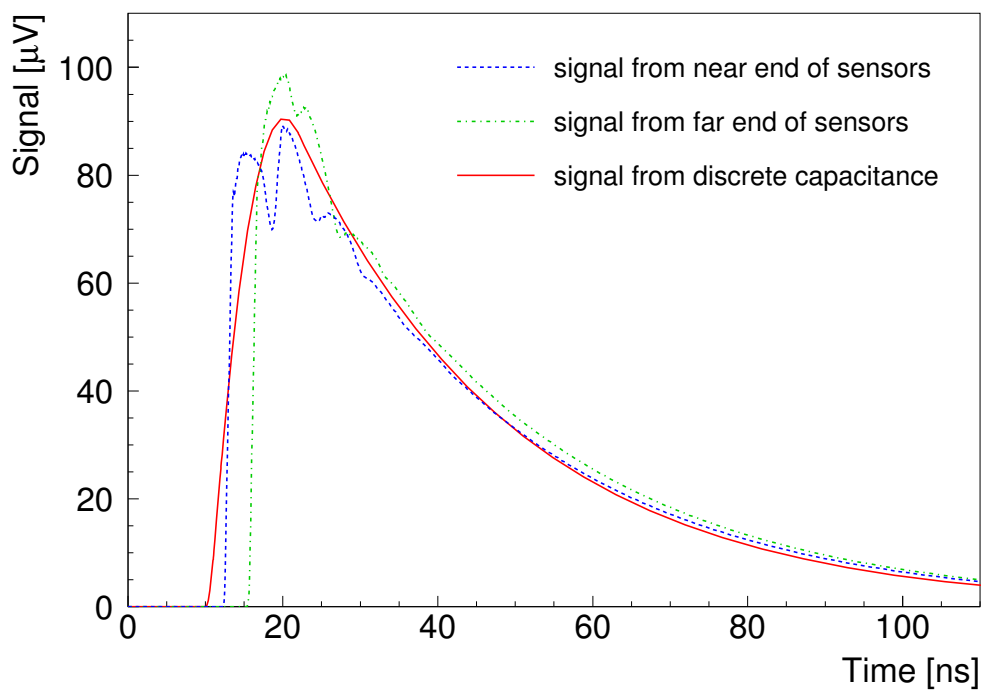

Figure 3: Simulated pulse shapes at the input of the Beetle front-end amplifier.



Figure 4: Simulated and measured pulse shapes at the output of the Beetle front-end amplifier.

teststand ${ }^{3}$.

The simulation shows that the pulse height for signals from the ladder is about $4 \%$ lower than that obtained for the equivalent discrete capacitance. The signal generated at the far end of the ladder arrives about $3 \mathrm{~ns}$ later than the signal generated at the end of the sensors closest to the front-end hybrid, i.e. close to the interface between sensors and interconnect cable. Such small differences are not expected to have a significant effect on the performance of the detector. In beam-tests, a uniform signal-to-noise performance was indeed measured

\footnotetext{
${ }^{3}$ The amplitude of the measured pulse was normalised to the amplitude of the simulated pulse. The discrepancy between simulation and measurement in the tail of the pulse is understood and is due to a simplification in the simulation of the Beetle chip. For details, see [7].
} 
over the full length of the prototype detector.

\section{Summary}

An extensive $R \& D$ program has been carried out to establish the expected signal-to-noise performance for the long detector ladders employed in the LHCb Silicon Tracker. Measurements and simulations indicate that the resistance and the inductance of the long readout strips have no significant effect on noise performance and pulse shape.

Noise performance, in general, depends on a close interplay of the noise frequency spectrum of the detector and the response spectrum of the readout amplifier. Therefore, the results obtained here cannot be easily generalised. Each detector configuration requires a dedicated study.

The obtained results demonstrate that a signal-to-noise performance in excess of 12:1 can be expected for all types of detector ladders employed in the Silicon Tracker. This should be sufficient to ensure fully efficient and robust operation of the detector over the full lifetime of the $\mathrm{LHCb}$ experiment [8].

\section{References}

[1] C. Parkes, proceedings of VERTEX 2005, to be published in Nucl. Instr. and Meth. A, and references therein

[2] J.-L. Agram et al., CMS note 2003-015

[3] N. Bakel et al., LHCb note 2005-105

[4] A. Gassner et al., LHCb note 2004-102

[5] M. Agari et al., LHCb notes 2004-103, 2003-082, 2002-085

[6] http://www.ansoft.com/products/em/max2d/

[7] S. Köstner and U. Straumann, LHCb note 2005-029

[8] M. Siegler et al., LHCb note 2004-070 